\title{
Healing pattern of the cervical stroma following cold coagulation treatment for cervical intraepithelial neoplasia: A case report
}

\author{
DIMITRIOS PAPOUTSIS $^{1,2}$, JOANNA WILLIAMS ${ }^{3}$, MARTYN UNDERWOOD $^{1}$ and WILLIAM PARRY-SMITH ${ }^{1}$ \\ ${ }^{1}$ Department of Obstetrics and Gynaecology, Shrewsbury and Telford Hospitals NHS Trust, Telford TF16TF, UK; \\ ${ }^{2}$ Midwifery Department, School of Health Sciences, University of Western Macedonia, 50200 Kozani, Greece; \\ ${ }^{3}$ Department of Histopathology, Shrewsbury and Telford Hospitals NHS Trust, Telford TF16TF, UK
}

Received August 2, 2021; Accepted November 5, 2021

DOI: $10.3892 / 01.2022 .13201$

\begin{abstract}
Cold coagulation of the cervix for cervical intraepithelial neoplasia (CIN), when compared with cervical excision, has previously demonstrated comparable cure rates and a reduction in the rate of spontaneous preterm birth. In the present report the healing pattern in the cervices of two women after cold coagulation is described. Both women underwent cold coagulation due to CIN3, which was found on pre-treatment cervical punch biopsies. They were followed up after cold coagulation and at 7 and 18 months, respectively, they underwent cervical excision. The histopathological slides from the excised specimen were reviewed, which represents the healed cervix after cold coagulation. A clear boundary of collagenisation was noted in the superficial stroma, which appeared to stop at the junction with the healthy muscular stroma. The collagenised superficial stroma depth, which represents the area that was thermally ablated and has now healed, measured 1.6 and $1.5 \mathrm{~mm}$ for the two women, respectively, which is less compared with that typically removed by cervical excision. Observations from these two cases indicate that cold coagulation does not appear to disrupt the deep tissue architecture of the cervix and could therefore explain the reduced levels of adverse obstetric morbidity in patients who underwent cold coagulation ablative treatment of the cervix, which has been previously reported.
\end{abstract}

\section{Introduction}

Cervical intraepithelial neoplasia (CIN) is an abnormality in the squamous cells of the cervix that, if left untreated, may progress to cervical cancer (1). This condition is asymptomatic and is histopathologically diagnosed when cervical punch

Correspondence to: Professor Dimitrios Papoutsis, Midwifery Department, School of Health Sciences, University of Western Macedonia, 50200 Kozani, Greece

E-mail: dpapoutsis@uowm.gr

Key words: cold coagulation, cervical intraepithelial neoplasia, healing, recurrence, obstetric morbidity biopsies are taken from women referred to colposcopy because of an abnormal cervical cytology test detected by screening (1). Depending on how many thirds of the thickness of the cervical surface layer are affected by the abnormal cells the severity of CIN is graded as CIN1, CIN2 or CIN3 (2). Treatment is usually offered to the patients when there is CIN 2 or 3 because there is a high chance of progression to cervical cancer (1).

Cold coagulation is a method of ablation to treat CIN lesions, and thus, prevent progression to cervical cancer. It is a method that was first introduced by Semm in 1966 in the United Kingdom (3). Using this method the cold coagulator probe is electrically heated to a temperature of $100-120^{\circ} \mathrm{C}$, which is then applied to the cervix for 20-45 sec per application, allowing for the ablation of cervical lesions $(4,5)$. Compared with excisional methods of cervical treatment, such as large loop excision of the transformation zone (LLETZ), there is evidence suggesting that cold coagulation has comparable cure rates $(4,5)$. In a systematic review and meta-analysis performed by Dolman et al (4) in 2014 involving 4,569 women from 13 studies who were treated with cold coagulation for CIN lesions, the cure rate was found to be $\sim 95 \%$ for CIN2+ lesions (4). In the updated systematic review and meta-analysis conducted by Randall et al (5) in 2018 with 6,371 women from 23 studies who were treated with cold coagulation for biopsy-proven CIN2+ lesions, the cure rate was $23.8 \%$ (5). In addition, there have been reports that have directly compared cold coagulation with LLETZ cervical treatment, which demonstrated comparable cytology and virological cure rates after treatment $(6,7)$.

Previous separate Cochrane database systematic reviews published in 2015 and 2017 have shown that excisional and ablative cervical treatment is associated with a significantly increased risk of miscarriage in the second trimester $(1,8)$. Furthermore, there is a higher risk of preterm birth for women treated using excisional methods compared with that in women who underwent ablative methods, with this risk increasing as the quantity of cervical tissue removed increases $(1,8)$. However, neither of these two previous systematic reviews managed to identify any studies of cold coagulation in the literature to include in the ablative methods arm of treatment. Other observational studies have nevertheless shown that cold coagulation when compared to cervical LLETZ treatment appeared to be more 'cervix friendly' in terms of obstetric 
outcomes in a future pregnancy, since it was reported to be associated with reduced risk of spontaneous preterm birth and miscarriage rates (9).

The biological mechanism that leads to increased spontaneous preterm birth and miscarriage rates after excisional and ablative cervical treatment remains unclear (10). It has been hypothesized that this may be associated with the lack of mechanical support due to a shortened cervix, changes in the immunological properties of the endocervix and/or alterations in the quality of the healed tissue of the cervix (11). Furthermore, there have been efforts to scientifically quantify the cervical dimensions after cervical treatment, which revealed that pregnancy outcomes after excisional treatment of the cervix are correlated with the proportion of cervix removed and the remaining cervical volume (12-14).

According to the literature, there has only been one study published in 2011 reporting the healing pattern of the cervix after excisional treatment for CIN lesions (15). This previous study had a small sample size $(n=17)$ and showed increased collagen expression in the regenerated cervical tissues of six women, reduced collagen expression in five women and an equivocal pattern of collagen distribution in six women (15). It was then concluded that there was no overall change in the collagen distribution during the regeneration process of the cervix following excisional treatment for CIN, but further research is necessary with a larger sample size (15).

In the present report, the healing pattern in the cervix after cold coagulation treatment in two women was presented. The objective was to map the collagenisation profile of the thermally-ablated cervix in relation to the healthy deep stromal structure of the cervix.

\section{Case report}

Cases. Case A is a 24-year-old nulliparous woman who is also a non-smoker and underwent her first ever cervical cytology screening test, which revealed severe dyskaryosis. In September 2014, she underwent colposcopy at the Shrewsbury and Telford Hospitals NHS Trust (Telford, UK), revealing a high grade colposcopic appearance of the cervix, following which two colposcopically-directed cervical punch biopsies were taken. The biopsies uncovered CIN3 and she underwent a single cold coagulation cervical treatment. Her first follow-up cytology test was at 6 months after treatment, which again demonstrated severe dyskaryosis. Therefore, she subsequently underwent LLETZ cervical treatment. The histopathology results of the cervical excision specimen showed CIN3, with dimensions of the excised tissue being 15x10x10 mm. The endocervical and deep lateral margins were clear, though complete excision at the ectocervical margin could not be confirmed. The patient is disease-free at follow-up ever since. The first follow-up cytology test (test of cure) was at 6 months after LLETZ excision and was cytology negative and HPV negative. The patient was then discharged to 3-yearly cytology screening. The last cytology screening test, which was negative, was in October 2021.

Case B is a 30 -year-old nulliparous woman who does not smoke and underwent a cytology screening test, showing severe dyskaryosis. In January 2014, she underwent colposcopy at the Shrewsbury and Telford Hospitals NHS Trust which showed that she had a high grade colposcopic appearance of the cervix, where subsequently two colposcopically-directed cervical punch biopsies were taken. Her biopsies also showed CIN3 and she underwent cold coagulation cervical treatment. Her first follow-up cytology test was scheduled at 6 months after treatment, but she was pregnant and her appointment was therefore deferred until 3 months after childbirth following a term pregnancy. Her cervical cytology screening test then revealed a negative result but was tested positive for high-risk human papilloma virus (HPV). She subsequently underwent colposcopy, demonstrating a low grade colposcopic appearance of the cervix before two cervical punch biopsies were taken revealing CIN3. She then had LLETZ cervical treatment at 18 months after her initial cold coagulation. The histopathology result of the cervical excision specimen showed CIN3, with dimensions of the excised tissue being $22 \times 20 \times 10 \mathrm{~mm}$. The endocervical margins were clear, but complete excision at the ectocervical margin could not be confirmed. The patient is disease-free at follow-up ever since. The first follow-up cytology test (test of cure) was at 6 months after LLETZ excision and was cytology negative and HPV negative. The patient was then discharged to 3 -yearly cytology screening. The last cytology screening test, which was negative, was in December 2018.

Both women were fully counselled on the risks and benefit of ablative compared with excisional techniques. Cold coagulation treatment was performed in accordance with the National Health Service Cervical Screening Programme guidance $(16,17)$. This means that cold coagulation was offered to women with no suspicion of cervical cancer according to their colposcopy examination results and those who had cervical punch biopsies taken prior to treatment. The colposcopy examinations and cervical treatments in these two women were undertaken by British Society of Colposcopy and Cervical Pathology (BSCCP) accredited colposcopists in the colposcopy unit of the Shrewsbury and Telford Hospitals NHS Trust in England (DP, MU and WPS). The Semm cold coagulator machine (model 60001; WISAP Medical Technology GmbH) was used and prior to treatment the cervix was injected with a local anesthetic (Citanest 3\% with Octapressin; $2.2 \mathrm{ml} / \mathrm{vial}$ ). The cold coagulator probe was then applied to the cervix with a temperature of $110-120^{\circ} \mathrm{C}$ for a minimum of $20 \mathrm{sec}$, where three applications were recorded per each woman.

Histopathological staining. The histopathological review of the slides was performed using $H \& E$, Sirius red and van Giessen staining. The cervical excision tissues of these two women were stored as wax blocks in routine storage at ambient temperature prior to staining. The fixation protocol involved the use of $10 \%$ neutral buffered formalin as the fixant, with the duration of fixation being between 24 and $36 \mathrm{~h}$ at room temperature. The tissues were embedded in paraffin. Dehydration was performed with 3x100\% xylene dips followed by $3 \times 100 \%$ alcohol dips to visibly clear the wax from the slide. $\mathrm{H} \& \mathrm{E}$ staining was performed on a Leica Autostainer XL (Leica Microsystems $\mathrm{GmbH}$ ) machine at room temperature throughout and according to the manufacturer's protocol. Normal light microscopy was used to image the H\&E staining at a magnification of $\mathrm{x} 2$ and $\mathrm{x} 4$.

In the van Giessen protocol, the tissue sections were fixed, embedded, sectioned, cleared (xylene and alcohol dips as 
A

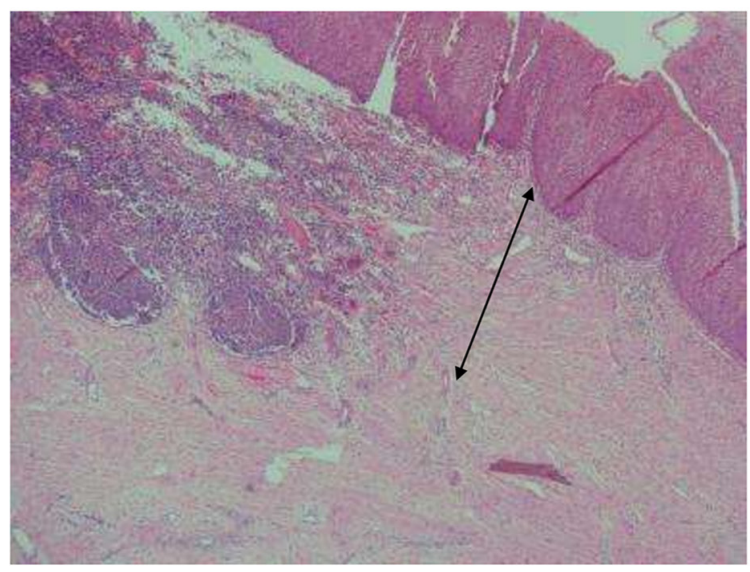

C

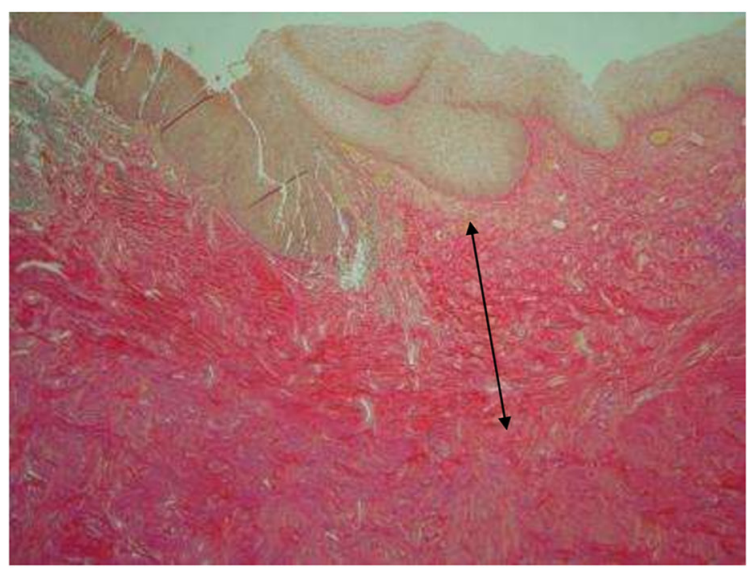

B

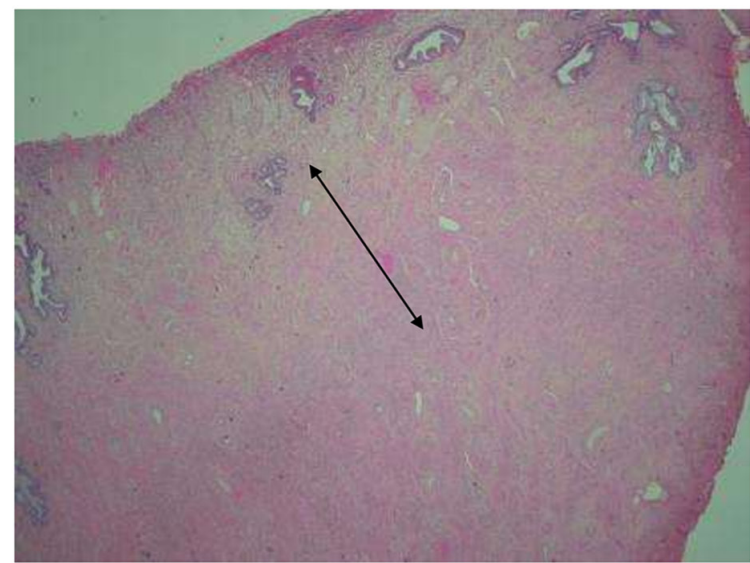

D

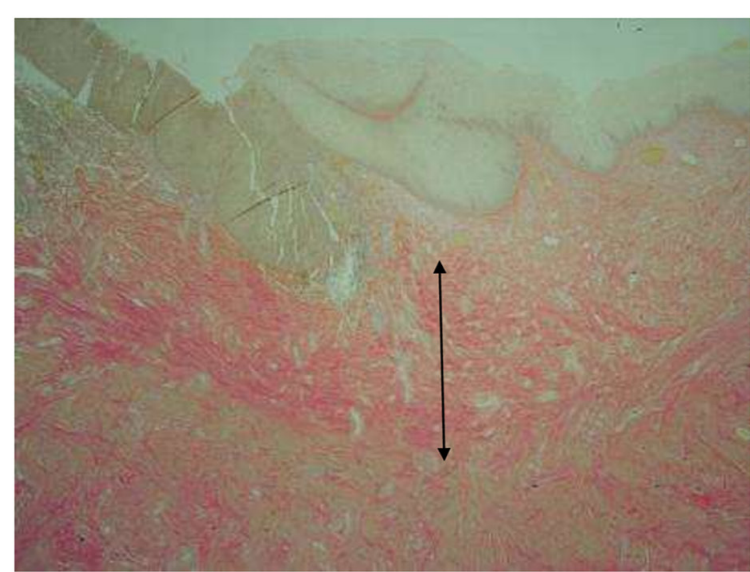

Figure 1. Histopathological slides from the excised cervical tissue specimen showing the healed cervix after the initial cold coagulation treatment. The healed collagenised area of the cervix is shown against the healthy deeper stroma. The arrows serve as indicators that depict the measurement of the collagenised cervix. (A) H\&E staining of the excised cervix from case A. Magnification, x4. (B) H\&E staining of the excised cervix from case B. Magnification, x2. (C) Sirius Red staining from case A. Magnification, x2. (D) Van Giessen staining from case A. Magnification, x2. The Sirius Red staining and van Giessen staining of the excised cervix from woman B are not shown, as these stains showed no significant difference between the thermally ablated areas and the healthy deeper stroma.

aforementioned) and then taken to distilled water. Then, they were treated with $4 \%$ aqueous ammonium iron (III) sulphate for $4 \mathrm{~min}$ at room temperature. They were subsequently washed thoroughly with running tap water and then rinsed with distilled water. They were stained with Mayers haematoxylin for $4 \mathrm{~min}$ at room temperature and then washed again in running tap water. They were differentiated with $1 \%$ acid alcohol at room temperature for a few seconds as dark staining happens quickly and is visible. Subsequently, it was checked microscopically that the nuclei were darkly stained and the background was clear. They were washed again in running tap water and rinsed with distilled water. Next, they were stained with Van Giesson's solution for $90 \mathrm{sec}$ at room temperature and washed with distilled water. Finally, they were rapidly dehydrated, cleared and mounted for microscopical review. Dehydration was performed with 3x100\% xylene dips followed by $3 \times 100 \%$ alcohol dips. Normal light microscopy was used to image the staining at a magnification of $\mathrm{x} 2$ and $\mathrm{x} 4$.

The Sirius red protocol initially involved de-waxing the paraffin sections. Dewaxing was performed with $3 \times 100 \%$ xylene dips followed by $3 \times 100 \%$ alcohol dips to visibly clear the wax from the slide. Next, the nuclei were stained with Weigerts haematoxylin for $8 \mathrm{~min}$ at room temperature and then washed for $10 \mathrm{~min}$. The sections were then stained for $1 \mathrm{~h}$ at room temperature in picro-sirius red solution (Sirius red F3B (C.I. 35782) $0.5 \mathrm{~g}$, saturated aqueous solution of picric acid $500 \mathrm{ml}$; Atom Scientific). Subsequently, they were washed in two changes of acidified water $(5 \mathrm{ml}$ acetic acid in 11 distilled water). Most of the water was physically removed from the slides by shaking and they were then dehydrated in three changes of $100 \%$ ethanol. Finally, the tissue sections were cleared in xylene and mounted for microscopical review. Normal light microscopy was used to image the Sirius red staining at a magnification of $\mathrm{x} 2$ and $\mathrm{x} 4$.

Clinical observations. Histopathological analysis showed a well-demarcated collagenisation of the superficial stroma, which appears to replace the more loosely organized superficial stroma and stop at the junction with the healthy muscular stroma (Fig. 1). For cases A and B, the collagenised superficial stroma depth, which represents the area that was thermally ablated and has now healed, measured 1.6 and $1.5 \mathrm{~mm}$, respectively. In addition, the normal healthy background superficial stroma depth was 1.8 and $3.5 \mathrm{~mm}$, respectively. The collagenisation pattern appeared to be similar between the two women. 


\section{Discussion}

The present report described two cases of cold coagulation treatment of the cervix due to CIN3, where the case A underwent subsequent LLETZ excision at 7 months after her initial ablative treatment whereas case B had LLETZ at 18 months after her initial ablative treatment, since she was pregnant in between. The excised cervical specimen represents the healed cervix, therefore histopathological analysis revealed information about the healing pattern after initial cold coagulation. To the best of our knowledge, the present study design has been implemented only once previously by Phadnis et al (15) in 2011, where a histopathological review was undertaken in women who underwent initial excisional treatment and then underwent another excisional treatment due to treatment failure or had a hysterectomy.

The present two cases show that cold coagulation appears to affect the superficial stroma of the cervix and does not disrupt the deeper stroma architecture. The collagenised superficial stroma depth, which represents the area that was thermally ablated but has now healed, measured 1.6 and $1.5 \mathrm{~mm}$, respectively. This is a depth that is lower compared with what is recommended to be removed by LLETZ. Current recommendations state that cervical excisions should be performed at the depth of $\geq 7 \mathrm{~mm}$ but $<10 \mathrm{~mm}$ in reproductive-aged women, since the endocervical glands extend to a maximum depth of $5.22 \mathrm{~mm}$ from the surface of the cervix (17-19). There have been reports that even though the colposcopist may opt for the recommended excision depth of $10 \mathrm{~mm},>50 \%$ women experienced actual excision depths that exceeded this limit $(9,20,21)$.

During the cold coagulation procedure no cervical tissue is removed $(4,22)$. Instead, it is thermally ablated with reports of an ablation depth of 4-7 $\mathrm{mm}(4,22)$. The two cases in the present report are preliminary histopathological findings in support of the notion that cold coagulation does not appear to physiologically disrupt the deep tissue architecture of the cervix. Therefore, this possibly explains the reduced rates of adverse obstetric morbidity of these patients treated because of CIN previously reported (9). It has been repeatedly reported that there is a 'dose-response' effect, where the less cervical tissue is removed, the lower the risk of obstetric morbidity in a future pregnancy $(23,24)$.

However, certain limitations should be considered regarding the findings in the present report. Although the collagenised superficial stroma depth measured 1.6 and $1.5 \mathrm{~mm}$ in the two cases, after the cervix is thermally ablated a thin, unquantifiable layer of tissue is always vaporized, which should be added to the thickness of the healed superficial stroma to provide the total ablation depth. In addition, the two cases in the present report were actually unsuccessful in terms of the treatment aim, similar to the findings of Phadnis et al (15). It is therefore possible that the deep cervical stroma remains unaltered whilst only the superficial cervical stroma is collagenized as a result of suboptimal thermal ablation to a shallow depth. As previously mentioned, a precise estimate of the total ablation depth is difficult due to the vaporization of the superficial layer of the cervix during the thermal ablation. However, cold coagulation can be performed in a highly standardized manner in the colposcopy unit in the present report, such that there is no reason to suspect that the treatment protocols for the two patients were inappropriate. In addition, the overall cytology cure rates in the present colposcopy unit after cold coagulation are comparable compared with those after LLETZ (6). In the two present cases there were three applications of the cold coagulator onto the ectocervix, which is the standard procedure frequently applied in the present colposcopy unit (6).

It has been previously shown in patients treated with cervical excision that CIN recurrence or treatment failure does not always depend on the endocervical depth of excision but other risk factors may play a role, such as the ectocervical margin involved $(20,25)$. A disadvantage of cold coagulation, similar to all other ablative methods, is that there is no cervical specimen to determine the quantity of cervical tissue removed and to examine the margins of the removed tissue (5). In the present two cases, it was known that following LLETZ excision the ectocervical margins of the excision were not clear but were involved by CIN, despite the relatively large ectocervical surface area excised. The area of ectocervix that was removed with LLETZ in the present two cases were $15 \times 10$ and $22 \times 20 \mathrm{~mm}$, respectively. This suggests that the lesion size was relatively widely spread on the ectocervix, which raises the hypothesis that it may have been the ectocervical component of the initial thermally-ablated lesion and not the endocervical depth that is the factor that led to recurrence. If this hypothesis is correct, then the ablation depth was appropriate and the deep cervical stromal tissue may not be disrupted by cold coagulation, thereby explaining the reduced risk of adverse obstetric outcomes in a future pregnancy compared with that after LLETZ excision.

Another previous report suggested that the 'crypt theory' could potentially explain the reason for treatment failure (26). This theory was based on a small case series of cervical excisions, where it was suggested that dysplastic cells entrapped deep within the crypts continuously undergo proliferation and initially remain undetectable by cytology and colposcopy before they invade the cervical stroma and surface much later (26). It has also been suggested that heavy cauterization of the cervical crater post excision may be another potential culprit of this entrapment (26). A similar mechanism may exist in cases of treatment failure after cold coagulation but further research is warranted to investigate this.

The ideal study design to be considered for future research would be to include women with a thermally-ablated cervix, with a test of cure that is cytology and HPV negative at follow-up to confirm that they are cured. This would indicate that an optimal thermally ablated depth was reached and then subsequently undergo hysterectomy for benign reasons. In the present case, the hysterectomy specimen will represent the healed cervix after the thermal ablation, where histopathological examination can then be used to determine the collagenisation pattern in the cervical stroma. In addition, a separate series of women with a first excisional cervical treatment could be added to serve as a reference group to identify any differences on the healing pattern in the cervix between cold coagulation and cervical excision.

\section{Acknowledgements}

Not applicable. 


\section{Funding}

No funding was received.

\section{Availability of data and materials}

The datasets used and/or analysed during the current study are available from the corresponding author on reasonable request.

\section{Authors' contributions}

DP conceived and designed this case report. DP, JW, MU and WPS collected the clinical data. JW performed the histopathological analysis and provided the related images. DP, JW, MU and WPS wrote the initial draft of the report and performed analysis and interpretation of data. DP and JW confirm the authenticity of all the raw data. All authors have read and approved the final version of the manuscript.

\section{Ethics approval and consent to participate}

Not applicable.

\section{Patient consent for publication}

The patients gave written informed consent for publication of the case details and associated images.

\section{Competing interests}

The authors declare that they have no competing interests.

\section{References}

1. Kyrgiou M, Athanasiou A, Kalliala IEJ, Paraskevaidi M, Mitra A, Martin-Hirsch PP, Arbyn M, Bennett P and Paraskevaidis E: Obstetric outcomes after conservative treatment for cervical intraepithelial lesions and early invasive disease. Cochrane Database Syst Rev 11: CD012847, 2017.

2. Fabrizii M, Moinfar F, Jelinek HF, Karperien A and Ahammer H: Fractal analysis of cervical intraepithelial neoplasia. PLoS One 9: e108457, 2014.

3. Semm K: New apparatus for the 'cold-coagulation' of benign cervical lesions. Am J Obstet Gynecol 95: 963-966, 1966.

4. Dolman L, Sauvaget C, Muwonge R and Sankaranarayanan R: Meta-analysis of the efficacy of cold coagulation as a treatment method for cervical intraepithelial neoplasia: A systematic review. BJOG 121: 929-942, 2014.

5. Randall TC, Sauvaget C, Muwonge R, Trimble EL and Jeronimo J: Worthy of further consideration: An updated meta-analysis to address the feasibility, acceptability, safety and efficacy of thermal ablation in the treatment of cervical cancer precursor lesions. Prev Med 118: 81-91, 2019.

6. Papoutsis D, Underwood M, Parry-Smith W and Panikkar J: Comparison of cure rates in women treated with cold-coagulation versus LLETZ cervical treatment for CIN2-3 on pretreatment cervical punch biopsies: A retrospective cohort study. Arch Gynecol Obstet 295: 979-986, 2017.

7. Tadesse WG, Oni AAA and Hickey KPW: Effectiveness of cold coagulation in treating high-grade cervical intraepithelial neoplasia: The human papillomavirus evidence of cure. J Obstet Gynaecol 39: 965-968, 2019.

8. Kyrgiou M, Mitra A, Arbyn M, Paraskevaidi M, Athanasiou A, Martin-Hirsch PP, Bennett P and Paraskevaidis E: Fertility and early pregnancy outcomes after conservative treatment for cervical intraepithelial neoplasia. Cochrane Database Syst Rev 2015: CD008478, 2015.
9. Papoutsis D, Underwood M, Parry-Smith W and Panikkar J: Early and late pregnancy outcomes in women treated with cold-coagulation versus LLETZ cervical treatment for cervical intraepithelial neoplasia; a retrospective cohort study. Arch Gynecol Obstet 297: 1015-1025, 2018.

10. Paraskevaidis E, Kyrgiou M and Martin-Hirsch P: Have we dismissed ablative treatment too soon in colposcopy practice? BJOG 114: 3-4, 2007.

11. Kyrgiou M, Mitra A, Arbyn M, Stasinou SM, Martin-Hirsch P, Bennett $\mathrm{P}$ and Paraskevaidis E: Fertility and early pregnancy outcomes after treatment for cervical intraepithelial neoplasia: Systematic review and meta-analysis. BMJ 349: g6192, 2014.

12. Founta C, Arbyn M, Valasoulis G, Kyrgiou M, Tsili A, Martin-Hirsch P, Dalkalitsis N, Karakitsos P, Kassanos D, Prendiville W, et al: Proportion of excision and cervical healing after large loop excision of the transformation zone for cervical intraepithelial neoplasia. BJOG 117: 1468-1474, 2010.

13. Papoutsis D, Daskalakis G, Antonakou A, Rodolakis A, Mesogitis S and Antsaklis A: Sonographic measurement of cervical volume in nonpregnant women using the geometric formula for a cylinder versus the three-dimensional automated virtual organ computer-aided analysis (vocal). J Clin Ultrasound 39: 322-328, 2011.

14. Kyrgiou M, Valasoulis G, Stasinou SM, Founta C, Athanasiou A, Bennett P and Paraskevadis E: Proportion of cervical excision for cervical intraepithelial neoplasia as a predictor of pregnancy outcomes. Int J Gynaecol Obstet 128: 141-147, 2015.

15. Phadnis SV, Atilade A, Bowring J, Kyrgiou M, Young MP, Evans H, Paraskevaidis E and Walker P: Regeneration of cervix after excisional treatment for cervical intraepithelial neoplasia: A study of collagen distribution. BJOG 118: 1585-1591, 2011.

16. Luesley D and Leeson S (eds): Colposcopy and Programme Management. Guidelines for the NHS Cervical Screening Programme. 2nd edition. NHSCSP Publication No. 20. NHS Sheffield, 2010.

17. NHSCSP: Colposcopy and Programme Management. Guidelines for the NHS Cervical Screening Programme. 3rd edition. NHSCSP Publication No. 20. NHSCSP, Sheffield, 2016.

18. Anderson MC and Hartley RB: Cervical crypt involvement by intraepithelial neoplasia. Obstet Gynecol 55: 546-550, 1980.

19. Byrom J, Douce G, Jones PW, Tucker H, Millinship J, Dhar K and Redman CW: Should punch biopsies be used when high-grade disease is suspected at initial colposcopic assessment? A prospective study. Int J Gynecol Cancer 16: 253-256, 2006.

20. Ang C, Mukhopadhyay A, Burnley C, Faulkner K, Cross P, Martin-Hirsch P and Naik R: Histological recurrence and depth of loop treatment of the cervix in women of reproductive age: Incomplete excision versus adverse pregnancy outcome. BJOG 118: 685-692, 2011.

21. Papoutsis D, Kandanearachchi P, Antonakou A, Tzavara C and Sahu B: A method to improve the accuracy between the presumed depth of excision and the actual depth of excision in women receiving LLETZ cervical treatment; a single-center, two-operator experience. Hippokratia 22: 113-121, 2018.

22. Haddad NG, Hussein IY, Blessing K, Kerr-Wilson $R$ and Smart GE: Tissue destruction following cold coagulation of the cervix. J Gynecol Surg 4: 23-27, 1988

23. Papoutsis D, Rodolakis A, Mesogitis S, Sotiropoulou M and Antsaklis A: Regeneration of uterine cervix at 6 months after large loop excision of the transformation zone for cervical intraepithelial neoplasia. BJOG 119: 678-684, 2012.

24. Castanon A, Landy R, Brocklehurst P, Evans H, Peebles D, Singh N, Walker P, Patnick J and Sasieni P, PaCT Study Group: Risk of preterm delivery with increasing depth of excision for cervical intraepithelial neoplasia in England: nested case-control study. BMJ 349: g6223, 2014.

25. Ghaem-Maghami S, Sagi S, Majeed G and Soutter WP: Incomplete excision of cervical intraepithelial neoplasia and risk of treatment failure: A meta-analysis. Lancet Oncol 8: 985-993, 2007.

26. Paraskevaidis E, Athanasiou A, Kalliala I, Batistatou A, Paraskevaidi M, Bilirakis E, Nasioutziki M, Paschopoulos M, Lyons D, Arbyn M, et al: Invasive cervical cancer following treatment of pre-invasive lesions: A potential theory based on a small case series. Eur J Obstet Gynecol Reprod Biol 264: 56-59, 2021. 